\title{
Insuficiência Cardíaca Crónica em Hospitalização Domiciliária
}

\section{Chronic Heart Failure in Home Hospitalization}

Paulina Mariano ${ }^{1}$, Soraia Silva ${ }^{1}$, Filipa Leitão ${ }^{1}$, Joana Carvalho ${ }^{1}$, Maria Eufémia Calmeiro², Isabel Antunes², Maria Eugénia André ${ }^{3}$

\section{RESUMO}

INTRODUÇÃO: A insuficiência cardíaca crónica é considerada, a nível mundial, a principal causa de internamento em doentes com idade superior a 65 anos. A Unidade de Hospitalização Domiciliária (UHD) foi criada com o objetivo de minimizar as complicações hospitalares, proporcionar maior qualidade de vida e atingir melhores resultados funcionais.

MATERIAL E MÉTODOS: Estudo retrospetivo de um ano de hospitalização domiciliária da Unidade Local de Saúde de Castelo Branco, destacando apenas doentes internados por descompensação da insuficiência cardíaca crónica.

RESULTADOS: Neste período foram internados 99 doentes, 23,3\% por descompensação da insuficiência cardíaca crónica. Eram do sexo masculino, 52,1\%. A idade média foi de 79,8 anos $( \pm 10,6)$. O tempo médio de internamento foi de 6,7 dias ( \pm 3$)$. As principais causas da insuficiência cardíaca foram: hipertensão arterial $(43,4 \%)$ e arritmias (30,4\%). O principal fator de descompensação foi infeções (69,5\%). Estiveram sob perfusão inicial de furosemida, 56,5\%. Evoluíram favoravelmente 95,6\% (n=22) dos doentes e 4,3\% ( $n=1)$ foi transferido para o internamento convencional. Segundo a escala de NEWS, 7,9\%-12,7\% dos doentes tinham risco de serem admitidos em unidade de cuidados intermédios/intensivos ou morrer entre 60 dias. $O$ índice de comorbilidades de Charlson foi de 3-10 pontos e a sobrevivência num período de 10 anos estimava-se em 0\%-77\%. Noventa dias após a alta, 69,5\% não regressaram à urgência nem foram reinternados.

1. Interno de Formação Específica de Medicina Interna, Unidade Local de Saúde de Castelo Branco, Castelo Branco, Portugal. 2. Assistente Hospitalar do Serviço de Medicina Interna, Unidade Local de Saúde de Castelo Branco, Castelo Branco, Portugal, 3. Diretora do Centro de Responsabilidade Integrada de Medicina Interna, Unidade Local de Saúde de Castelo Branco, Castelo Branco, Portugal, 
CONCLUSÃO: A hospitalização domiciliária em doentes com insuficiência cardíaca é vantajosa por proporcionar maior conforto ao doente e reduzir as complicações. Estudos demonstraram que a hospitalização domiciliária aumentou o tempo entre as readmissões, melhorou a qualidade de vida dos doentes e reduziu os custos das hospitalizações.

PALAVRAS-CHAVE: Doença Crónica; Insuficiência Cardíaca; Qualidade de Vida; Serviços Hospitalares de Assistência Domiciliar

\section{ABSTRACT}

INTRODUCTION: Chronic heart failure (CHF) is considered, worldwide, the main cause for hospitalization in patients over the age of 65 years old. The Home Hospitalization Unit (HHU) was created in order to minimize hospital complications, to provide a better quality of life and to achieve better functional results.

MATERIAL AND METHODS: Retrospective study of one year of home hospitalization (HH) at the Local Health Unit in Castelo Branco, focusing only in hospitalized patients due to chronic heart failure decompensation.

RESULTS: During this period, 99 patients were hospitalized, 23.3\% due to decompensated heart failure). Were male, 52.1\%. The average age was 79.8 years old ( \pm 10.6$)$. The average hospital stay was 6.7 days ( \pm 3$)$. The main causes of heart failure were hypertension (43.4\%) and arrhythmias (30.4\%). The main factor for decompensation was infections (69.5\%). Were under initial infusion of furosemide, $56.5 \%$ of the patients. Evolved favorably $95.6 \%(n=22)$ of the patients and one (4.3\%) was transferred to conventional hospitalization. According to the NEWS scale, 7.9\%-12.7\% of the patients were at risk of being admitted to an intermediate/intensive care unit or at risk of dying within 60 days. Charlson's comorbidity index was 3-10 points and survival in a subsequent 10-year period was estimated at 0\%-77\%. Ninety days after discharge, $69.5 \%$ of the patients did not return to the emergency department neither were they readmitted.

CONCLUSION: Home hospitalization in patients with HF is advantageous because it provides greater comfort to the patient and reduces complications. Studies have shown that HH increased the time between readmissions, improved patients' quality of life and reduced hospitalization costs.

KEYWORDS: Chronic Disease; Heart Failure; Home Care Services, Hospital-Based; Quality of Life

\section{INTRODUÇÃO}

A insuficiência cardíaca é uma patologia com elevada morbi-mortalidade. Sendo uma doença crónica, terá períodos de remissões e agudizações. Em cada agudização há um declínio do estado geral que levará a hospitalizações cada vez mais frequentes. É considerada a principal causa de internamento em doentes com idade superior a 65 anos. $^{1}$

À medida que a população envelhece, a incidência e a prevalência de doenças crónicas aumentam, aumentando as hospitalizações e as despesas de saúde. Em 2030 estima-se um aumento de $46 \%$ da prevalência da insuficiência cardíaca, comparativamente ao ano 2012. Mais de $70 \%$ do orçamento anual dos cuidados aos doentes com insuficiência cardíaca são gastos em internamentos. ${ }^{1,2}$

As Unidades de Hospitalização Domiciliária (UHD) foram criadas como modelo de assistência hospitalar praticado no domicílio por um período transitório em alternativa ao internamento hospitalar convencional. ${ }^{2}$ Este conceito surgiu pela primeira vez em 1947, nos Estados Unidos da América, com a experiência "Home Care", com o objetivo de aumentar o número de camas nos hospitais, assim como criar um ambiente psicológico mais favorável para a recuperação do doente. ${ }^{3}$ A primeira UHD na Europa, surgiu em França, em 1951. Em Portugal o início das UHD foi em 2015 no Hospital Garcia da Horta, ${ }^{4}$ e 4 anos depois nasceu o projeto na ULS de Castelo Branco.

A hospitalização domiciliária (HD) permite que o doente receba cuidados na sua própria casa, iguais em qualidade e quantidade aos prestados no hospital, quando o doente necessita de cuidados mais diferenciados e que não tem condições para tratamento em regime ambulatorial, reunindo assim os critérios clínicos, sociais e geográficos, ${ }^{3}$ mas sempre de acordo com a vontade do doente e da sua família.

A UHD serve também de meio de educação para saúde e ensino dos vários pontos a abordar na gestão da ICC. 


\section{MATERIAL E MÉTODOS}

A UHD da ULS de Castelo branco foi criada em março de 2019. Desde 1 de abril de 2019 a 31 de março de 2020, foram internados em regime de hospitalização domiciliária doentes de várias idades e comorbilidades.

Foi feito um estudo retrospetivo com avaliação dos processos clínicos e análise em Excel.

Fez-se revisão bibliográfica de artigos que abordavam a insuficiência cardíaca crónica em regime de hospitalização domiciliária.

\section{RESULTADOS}

Neste primeiro ano de hospitalização domiciliária, foram internados 99 doentes, dos quais, 23,3\% ( $n=23)$ foi por insuficiência cardíaca descompensada. A prevalência foi ligeiramente maior no sexo masculino, 52,1\%, $n=12$ (sexo feminino 47,8\%, n=11). A idade variou entre os 62 e 96 anos, com uma média de 79,8 anos $( \pm 10,6)$. Quanto a proveniência, 73,9\% ( $n=17$ ) foram referenciados diretamente do serviço de urgência, 21,7\% ( $n=5)$ do internamento de Medicina e 4,3\% ( $n=1)$ da consulta externa. $O$ tempo médio de internamento foi de 7,4 dias $( \pm 4,6)$.

Dos doentes internados por descompensação da insuficiência cardíaca, 73,9\% (n=17) tinham fração de ejeção preservada, três (13\%) a fração era intermédia e os restantes três (13\%) tinham a fração de ejeção reduzida. Do ponto de vista etiológico, as principais causas de insuficiência cardíaca foram: hipertensão arterial (43,4\%, $n=10)$, arritmias $(30,4 \%, n=7)$, patologia valvular $(13 \%$, n=3) e isquémica (13\%, n=3) (Fig. 1).

Os principais fatores que levaram a descompensação da insuficiência cardíaca foram: infeção (respiratória/ urinária, $n=16,69,5 \%)$; má adesão terapêutica ( $n=2,8,6 \%$ ); anemia ( $n=1,4,3 \%)$; síndrome coronária aguda $(n=1$, 4,3\%); em três dos doentes (13\%), não foi identificada a causa (Fig. 2).

Das várias comorbilidades neste grupo de doentes destacam-se: hipertensão arterial ( $n=14,60,8 \%)$, diabetes mellitus tipo 2 ( $n=11,47,8 \%)$, fibrilação auricular ( $n=8$, 34,7\%; três dos quais eram portadores de pacemaker), dislipidémia ( $n=8,34,7 \%)$, doença renal crónica ( $n=5$, $21,7 \%)$ e outras ( $n=7,30,4 \%$ ) nomeadamente, obesidade, síndrome da apneia obstrutiva do sono, hipertiroidismo, leucemia mieloide aguda e doença pulmonar obstrutiva crónica (Fig. 3).

Segundo o índice de Barthel, 60,8\% ( $n=14)$ dos doentes eram autónomos, $26 \%(n=6)$ parcialmente dependentes e $13 \%(n=3)$ totalmente dependentes nas atividades de vida diária.

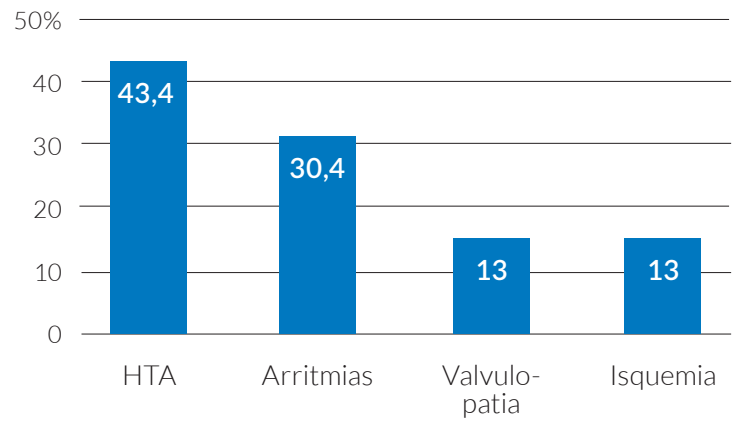

FIGURA 1. Distribuição percentual das principais causas de insuficiência cardíaca.

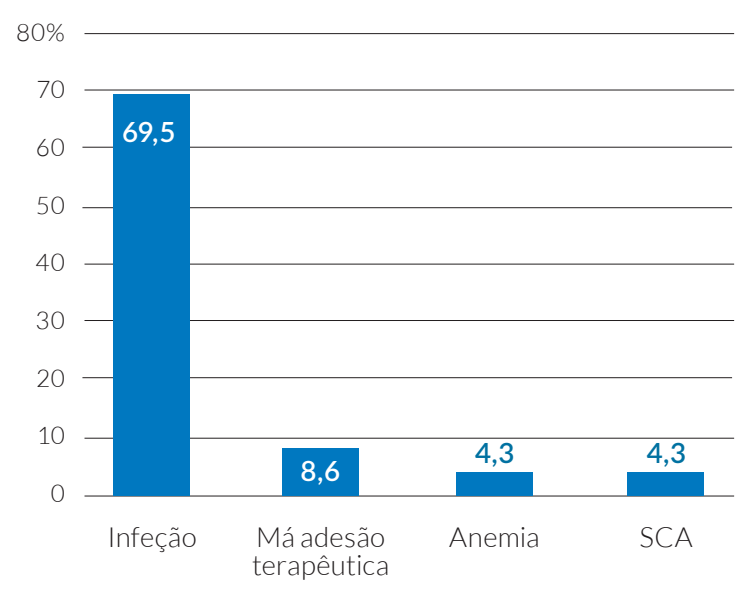

FIGURA 2. Distribuição percentual dos principais fatores de descompensação da insuficiência cardíaca. SCA (síndrome coronária aguda).

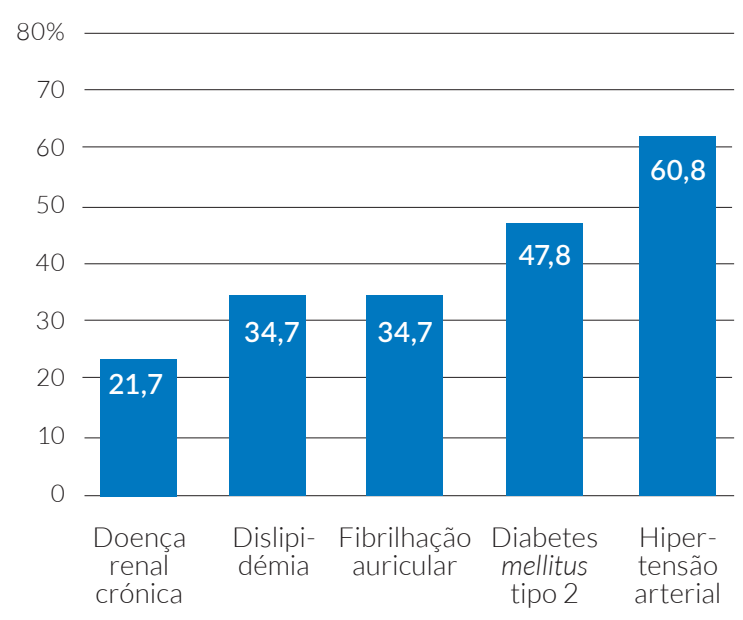

FIGURA 3. Distribuição percentual das principais comorbilidades dos doentes com insuficiência cardíaca.

Os doentes no início do internamento estavam muito sintomáticos (NYHA III-IV). A maior parte necessitou de altas doses de diuréticos (56,5\%) e por isso estiveram sob perfusão contínua de furosemida, 43,4\% ( $n=10)$ 
fizeram bólus, para além da outra terapêutica. Houve melhoria franca dos sintomas (NYHA I-II a data da alta). Durante o internamento foi monitorizado o peso dos doentes e observou uma redução do peso (média à entrada 79, à saída 75 kg).

Quanto às intercorrências observadas durante o internamento, as principais foram: lesão renal (crónica agudizada ou aguda) 39,1\%, hipocalemia 21,7\%, agravamento da dispneia (13\%), crise hipertensiva (4,3\%) e agitação psicomotora (4,3\%).

De acordo com a escala de NEWS (National Early Warning Score), que avalia o estado de deterioração clínica em contexto hospitalar e não só, os doentes internados tiveram um risco de 7,9\%-12,7\% (1-3 pontos) de serem admitidos em unidade de cuidados intermédios, intensivos ou morrer entre 60 dias. Quanto ao índice de comorbilidades de Charlson, os doentes tiveram 3-10 pontos, isto é, a sobrevivência em 10 anos estimava-se em 0\%-77\%.

Dos 23 doentes internados, 95,6\% (n=22) evoluíram favoravelmente. Um dos doentes (4,3\%) foi transferido para o internamento convencional.

Reavaliando os processos dos utentes 90 dias após a alta, os resultados foram bons, sendo que, 69,5\% ( $n=16)$ não regressaram ao serviço de urgência nem foram reinternados (Medicina ou noutros serviços); seis (26\%) regressaram ao serviço de urgência, dos quais, 5 (83,3\%) foi por sintomatologia não associada à insuficiência cardíaca e apenas um tinha queixas de insuficiência cardíaca (por incumprimento terapêutico, mas sem necessidade de internamento). Não se registou nenhum reinternamento.

Foi avaliado o grau de satisfação dos doentes, familiares ou cuidadores através do preenchimento de um questionário. Estavam muito satisfeitos 91,3\% e 8,6\% satisfeitos com o trabalho realizado pela equipa de HD.

\section{DISCUSSÃO}

A insuficiência cardíaca crónica é uma patologia com recorrentes períodos de agudizações. A incidência de internamentos por descompensação de insuficiência cardíaca triplicou em menos de três décadas. Nos países desenvolvidos é considerada a principal causa de internamento em indivíduos com mais de 65 anos, representando um fardo económico significativo para o sistema nacional de saúde. Doentes internados são mais suscetíveis a complicações, aumentando assim, o risco de morte e reduzindo a qualidade de vida. ${ }^{1}$
A prevalência das doenças crónicas aumenta com a idade. Visto que a tendência destas doenças é progredir ao longo dos anos, haverá maior necessidade de internamentos e deste modo aumentarão os custos relacionados com a saúde. Apesar da otimização terapêutica, a probabilidade de metade destes doentes serem reinternados entre 6 meses após a alta, é muito elevada, causando declínio funcional, iatrogenia, maior risco de infeções assim como, maiores custos sanitários. ${ }^{1,2}$

A hospitalização domiciliária é uma prestação de cuidados de nível hospitalar no domicílio do doente, como alternativa do internamento convencional, de modo a melhorar a qualidade de vida, proporcionar maior conforto ao doente, familiar ou cuidador e adquirir melhores resultados funcionais. ${ }^{1}$ Doentes com critérios para admissão hospitalar podem ser propostos para internamento em casa, em nenhuma circunstância se poderá prejudicar o doente por se encontrar internado no domicílio e serão prestados cuidados iguais em qualidade e quantidade aos prestados no hospital. ${ }^{3-5}$

Este regime de internamento, segundo a literatura tem muitas vantagens: aos doentes, familiares ou cuidadores, proporciona melhor atenção, participação e responsabilidade; melhor qualidade de vida e bem-estar, assistência personalizada, oportunidade de educação para saúde e menor risco de iatrogenia. A nível hospitalar, maior rentabilidade dos recursos disponíveis, maior rotação e disponibilidades de camas, redução do período de internamento; no sistema nacional de saúde, aumenta a eficácia e eficiência mediante assistência mais humanizada e uso mais eficaz dos recursos. ${ }^{3}$

A equipa de hospitalização domiciliária está incorporada no serviço de Medicina Interna da ULS de Castelo Branco. Os doentes foram referenciados do serviço de urgência, do internamento de Medicina e da Consulta Externa. Após referenciação a equipa de hospitalização domiciliária avaliava os doentes, verificando se reuniam os critérios clínicos, sociais e geográficos e definia-se o protocolo terapêutico. As visitas domiciliárias eram programadas em função de necessidade de cada doente, podiam ser singulares (enfermeiro) e interdisciplinares (médico e enfermeiro).

○ primeiro ano de hospitalização domiciliária foi um grande desafio, tanto para os profissionais de saúde como para os doentes, familiares ou cuidadores. Dos doentes internados por insuficiência cardíaca descompensada, a prevalência foi quase sobreponível em ambos os sexos e 73,9\% ( $n=17)$ tinham uma idade superior ou igual a 75 anos. Portanto, eram utentes idosos, denominados frágeis pelas várias comorbilidades e poli- 
medicação. É um grupo complexo de cuidar, para além da descompensação das várias patologias de base, em internamento hospitalar têm maior risco de iatrogenia, delírio e adquirir infeções.

Várias são as patologias que podem desencadear a insuficiência cardíaca; no grupo dos doentes internados as principais foram: hipertensão arterial e arritmias. Quanto às causas de descompensação, mais frequentemente cursam com um ou mais fatores, tais como infeção, hipertensão não controlada, distúrbios do ritmo ou a não adesão a fármacos/dieta; mas pode ocorrer sem fatores precipitantes conhecidos.

Foi usado o índice de Charlson para identificar o tipo de doentes consoante as comorbilidades. Este índice avalia o risco de morte e mede a gravidade da doença, utilizando morbilidades com diferente impacto no prognóstico. A pontuação varia de 1-6 (exemplo: 6 tumor sólido metastizado, diabetes mellitus sem e com complicações "1 e 2 pontos"). Dependendo do tipo e número de comorbilidades, estima-se a mortalidade (exemplo: $\geq 3$ pontos significa grave comorbilidade). ${ }^{6}$ Aplicando este índice nos doentes internados em hospitalização domiciliária, a pontuação obtida foi de 3-10 pontos, o que significa que os doentes tinham graves comorbilidades e a sobrevivência em 10 anos estimava-se em 0\%-77\% (ajustada a idade). Portanto, eram doentes com muitas comorbilidades, com elevado risco de complicações e de mortalidade.

A escala NEWS é considerada a nível mundial, uma das melhores ferramentas para avaliar o grau de risco dos doentes (degradação clínica) tanto em contexto hospitalar como no pré-hospitalar. Avalia a frequência respiratória, saturação de oxigénio, necessidade de oxigénio, temperatura, pressão arterial sistólica, frequência cardíaca e o estado de consciência. A pontuação varia de 0 (parâmetros normais) a 3 (alterações mais graves, por exemplo: hipotermia/ hipertermia, bradicardia/ taquicardia, etc.). ${ }^{7}$ Os doentes internados tiveram um risco estimado de 7,9\%-12,7\% de serem admitidos em unidade de cuidados intermédios, intensivos ou morrer entre 60 dias. Isto significa que eram doentes que apresentavam uma certa gravidade, com critérios de internamento, mas que estavam estáveis para serem tratados em regime domiciliário.

Durante o internamento foram evidenciadas algumas intercorrências, associadas a insuficiência cardíaca ou ao tratamento instituído. São elas, a deterioração da função renal, hipocalemia, agravamento da dispneia, hipertensão arterial não controlada e agitação psicomotora.
A hospitalização domiciliária é particularmente fundamental neste grupo de doentes, porque estando no domicílio, previnem-se as intercorrências hospitalares, proporcionando melhor qualidade de vida e bem-estar aos utentes. Todas estas atitudes influenciam na redução da mortalidade. Este regime de internamento é também vantajoso porque exige maior envolvimento dos doentes/familiares/ cuidadores no manuseio da medicação e desta forma compreendem melhor a doença e pode-se reforçar a importância do cumprimento terapêutico e da dieta nestes doentes. Surge assim como janela de oportunidade para a educação terapêutica identificando os problemas dentro da própria casa do doente, nas suas vivências e tenta-se corrigir no seu meio. Percebem-se as limitações e a hospitalização domiciliária serve de ponte para reforçar as atitudes.

A maior parte dos doentes tiveram uma evolução clínica favorável com alta para as consultas. Reavaliando os processos dos utentes 90 dias após a alta, os resultados foram bons, sendo que, 69,5\% não regressaram ao serviço de urgência nem foram reinternados. Dos poucos que regressaram ao serviço de urgência, apenas um tinha queixas de insuficiência cardíaca por incumprimento terapêutico, mas não necessitou de ser internado. Não houve registo de nenhum reinternamento.

Quanto ao grau de satisfação baseado no questionário que os doentes, familiares ou cuidadores preencheram, pode-se afirmar que todos ficaram satisfeitos com o trabalho realizado pela equipa de HD.

\section{CONCLUSÃO}

Sendo a insuficiência cardíaca uma doença crónica, o curso da doença é caracterizado por períodos de agudizações com declínio progressivo do estado geral e necessidade de internamentos frequentes. A maior parte dos doentes internados foram idosos, com múltiplas comorbilidades e polimedicados. A hospitalização domiciliária é vantajosa nos doentes em geral, mas neste grupo em particular, pela melhoria da qualidade de vida, proporciona maior conforto e melhores resultados funcionais, evitando deste modo as complicações intra-hospitalares. A literatura sobre a abordagem da insuficiência cardíaca em hospitalização domiciliária é ainda muito limitada e baseada em pequenos estudos que carecem de mais investigações. Apesar das limitações, a hospitalização domiciliária demonstrou aumentar o tempo entre as readmissões, melhorou a qualidade de vida dos doentes e reduziu os custos sanitários. Quanto ao reduzir significativamente o número de readmissões ou a mortalidade, são necessários mais estudos randomizados. 
Em Portugal a UHD demonstrou ser uma ferramenta importante na gestão dos doentes com ICC e uma alternativa ao internamento convencional. Neste primeiro ano de experiência de hospitalização domiciliária, pode afirmar-se que foram cumpridos os indicadores de qualidade e desempenho preconizados a nível internacional.

\section{AGRADECIMENTOS/ACKNOW- LEDGMENTS}

Os autores agradecem à equipa de enfermagem da hospitalização domiciliária, nomeadamente, Emanuel Pereira, João Domingos, Catarina Marques, Hugo Caldeira, Filipa Amaro e Carlos Almeida, pela dedicação.

\section{RESPONSABILIDADESÉTICAS}

CONFLITOS DE INTERESSE: Os autores declaram a inexistência de conflitos de interesse na realização do presente trabalho.

FONTES DE FINANCIAMENTO: Não existiram fontes externas de financiamento para a realização deste artigo.

CONFIDENCIALIDADE DOS DADOS: Os autores declaram ter seguido os protocolos da sua instituição acerca da publicação dos dados de doentes.

PROTEÇÃO DE PESSOAS E ANIMAIS: Os autores declaram que os procedimentos seguidos estavam de acordo com os regulamentos estabelecidos pelos responsáveis da Comissão de Investigação Clínica e Ética e de acordo com a Declaração de Helsínquia da Associação Médica Mundial.

PROVENIÊNCIA E REVISÃO POR PARES: Não comissionado; revisão externa por pares.

\section{ETHICAL DISCLOSURES}

CONFLICTS OF INTEREST: The authors have no conflicts of interest to declare.

FINANCING SUPPORT: This work has not received any contribution, grant or scholarship

CONFIDENTIALITY OF DATA: The authors declare that they have followed the protocols of their work center on the publication of data from patients.

PROTECTION OF HUMAN AND ANIMAL SUBJECTS: The authors declare that the procedures followed were in accordance with the regulations of the relevant clinical research ethics committee and with those of the Code of Ethics of the World Medical Association (Declaration of Helsinki).
PROVENANCE AND PEER REVIEW: Not commissioned; externally peer reviewed.

\section{REFERÊNCIAS}

1. Qaddoura A, Yazdan-Ashoori P, Kabali C, Thabane L, Haynes RB, Connolly SJ, et al. Efficacy of Hospital at Home in Patients with Heart Failure: A Systematic Review and Meta-Analysis. PLoS One. 2015;10:e0129282. doi: 10.1371/journal. pone.0129282.

2. Voudris KV, Silver MA. Home hospitalization for acute decompensated heart failure: opportunities and strategies for improved health outcomes. Healthcare. 2018;6:31. doi: 10.3390/ healthcare6020031.

3. Cotta RM, Suárez-Varela MM, Llopis González A, Cotta Filho JS, Ramón Real E, Días Ricós JÁ. La hospitalización domiciliaria: antecedentes, situación actual y perspectivas. Rev Panam Salud Publica. 2001: 10.

4. Cunha V, Escarigo MC, Correia J, Nortadas R, Azevedo PC, Beirão P, et al. Hospitalização Domiciliária: Balanço de um Ano da Primeira Unidade Portuguesa Med Intern. 2017;24:290-5.

5. Direção Geral da Saúde. Hospitalização Domiciliária em idade adulta; Norma n 020/2018 de 20/12/2018. Lisboa:DGS; 2018.

6. Broeiro Gonçalves P, Nogueira P, Aguiar P. Multimorbilidade e Gravidade da Doença Medida pelo Índice de Charlson em Doentes Hospitalizados Durante o Ano de 2015: Estudo Transversal. Acta Med Port. 2019:32:38-46. doi: 10.20344/ amp.9728.

7. Leandro Luís, Tradução, validação e aplicação dos sistemas de pontuação de alerta precoce "VIEWS" e "NEWS" em Portugal. $2^{\circ}$ Curso de Mestrado de Gestão e Avaliação de Tecnologias da Saúde; Lisboa 2014. 